\title{
Interdisciplinary consensus statement on indication and application of a hydrogel spacer for prostate radiotherapy based on experience in more than 250 patients
}

\author{
Arndt-Christian Müller ${ }^{1}$, Johannes Mischinger ${ }^{2}$, Theodor Klotz ${ }^{3}$, Bernd Gagel ${ }^{4}$, \\ Gregor Habl ${ }^{5}$, Gencay Hatiboglu ${ }^{6}$, Michael Pinkawa ${ }^{7}$ \\ ${ }^{1}$ Department of Radiation Oncology, Eberhard Karls University, Tübingen, Germany \\ 2 Department of Urology, Eberhard Karls University, Tübingen, Germany \\ ${ }^{3}$ Department of Urology, Hospital Weiden, Weiden, Germany \\ ${ }^{4}$ Department of Radiation Oncology, Hospital Weiden, Weiden, Germany \\ ${ }^{5}$ Department of Radiation Oncology, Technical University Munich, Munich, Germany \\ ${ }^{6}$ Department of Urology, University of Heidelberg, Heidelberg, Germany \\ ${ }^{7}$ Department of Radiation Oncology, RWTH Aachen University, Aachen, Germany
}

Radiol Oncol 2016; 50(3): 329-336.

Received 28 February 2016

Accepted 17 April 2016

Correspondence to: Arndt-Christian Müller, M.D., Department of Radiation Oncology, Eberhard-Karls-University, 72076 Tübingen, Germany. Phone: +49 7071298 2165; Fax: +49 7071295 026; E-mail: arndt-christian.mueller@med.uni-tuebingen.de

Disclosure: No potential conflicts of interest were disclosed.

Background. The aim of the study was to reach a consensus on indication and application of a hydrogel spacer based on multicentre experience and give new users important information to shorten the learning curve for this innovative technique.

Methods. The interdisciplinary meeting was attended by radiation oncologists and urologists, each with experience of $23-138$ hydrogel injections (SpaceOAR®) in prostate cancer patients before dose-escalated radiotherapy. User experience was discussed and questions were defined to comprise practical information relevant for successful hydrogel injection and treatment. Answers to the defined key questions were generated. Hydrogel-associated side effects were collected to estimate the percentage, treatment and prognosis of potential risks.

Results. The main indication for hydrogel application was dose-escalated radiotherapy for histologically confirmed low or intermediate risk prostate cancer. It was not recommended in locally advanced prostate cancer. The injection or implantation was performed under transrectal ultrasound guidance via the transperineal approach after prior hydrodissection. The rate of injection-related G2-toxicity was $2 \%(n=5)$ in a total of 258 hydrogel applications. The most frequent complication $(n=4)$ was rectal wall penetration, diagnosed at different intervals after hydrogel injection and treated conservatively.

Conclusions. A consensus was reached on the application of a hydrogel spacer. Current experience demonstrated feasibility, which could promote initiation of this method in more centres to reduce radiation-related gastrointestinal toxicity of dose-escalated IGRT. However, a very low rate of a potential serious adverse event could not be excluded. Therefore, the application should carefully be discussed with the patient and be balanced against potential benefits.

Key words: prostate cancer; hydrogel spacer; dose-escalated radiotherapy; proctitis; toxicity 


\section{Background}

Dose escalated intensity-modulated radiation treatment (IMRT with radiation doses $\geq 76 \mathrm{~Gy}$ ) is a highly effective, curative treatment option for localized prostate cancer. Biochemical control is directly related to radiation dose with a dose effect per each additional Gy. ${ }^{1}$ For example, escalation from 70 to $80 \mathrm{~Gy}$ is connected with a $15 \%$ increase in PSA control. This dose effect is described for all risk groups. However, an increased radiation dose is also associated with rising levels of grade $\geq 2$ acute and chronic toxicity. ${ }^{1}$ Lower gastrointestinal toxicity rates can result from smaller posterior safety margins or even no safety margins ${ }^{2}$, potentially compromising local tumour control.

A novel method to reduce rectal toxicity during dose-escalated IMRT is the insertion of a hydrogel spacer between the Denonvilliers' fascia and anterior rectal wall to separate these structures. ${ }^{3}$ The created space generates a distance of $10-15 \mathrm{~mm}$ between both organs. ${ }^{4-6}$ Recent studies unequivocally demonstrated a significant reduction in high-dose areas on the anterior rectal wall. $4,5,7,8$ As expected, better rectal sparing from higher radiation doses was associated with only mild toxicity from the dose-escalated treatment. ${ }^{4,9,10}$

The application technique ${ }^{3}$, dosimetric studies $^{4,8}$ and some early toxicity data ${ }^{4,10}$, as mentioned above, were all published within the last two years. However, despite rising numbers of hydrogel injections, reports on practical aspects or pitfalls of hydrogel application as well as frequency and management of side effects of the administration were not or were only provided for single cases..$^{11}$ Therefore, the first consensus meeting was held in July 2013 to discuss this practical issue and to generate answers for users on the indication, application and management of side effects of a hydrogel spacer for dose-escalated radiotherapy. Thereafter, toxicity data of the injection technique was collected from $>250$ patients of four centres to better balance the benefit and potential risks of this new method.

The aim of this consensus report is to offer new users of this technique easy access to relevant information on practical application and patient management to shorten the learning curve ${ }^{7}$ and to carefully balance potential benefits against potential risks of this technique.

\section{Patients and methods}

The interdisciplinary meeting was attended by radiation oncologists and urologists, each with experi- ence of $23-138$ hydrogel injections (SpaceOAR®) in prostate cancer patients before dose-escalated IMRT. In the first part of the meeting, user experiences were discussed and questions were defined to comprise practical information relevant for successful gel injection and treatment. In the second part, answers to the defined key questions were developed. Prospective data from the multiinstitutional clinical trial ${ }^{10}$, prospective mono-institutional data (German Clinical Trials Register DRKS00003273) 4 and data collected retrospectively from patient files were considered in this interdisciplinary process to evaluate hydrogel application in current practice. The prospective studies were approved by each institution's ethics committee. With regard to the participating centres approvals were given by the University of Aachen ${ }^{10}$, the University of Heidelberg ${ }^{10}$ and the University of Tübingen. ${ }^{4}$ All of these patients $(n=62)$ gave their written informed consent to participate in these studies. ${ }^{4,10}$ After discussing the intended analysis of retrospectively collected data $(n=196)$ the institutional review board (Ethics Committee of the University of Tübingen) had no objections (266/2015BO2). Patients gave informed consent to standardized data documentation and evaluation of treatment related toxicity.

After the meeting, participants were asked to state the incidence of side effects to better balance risks and beneficial effects. Finally, the statement was revised and consented. Recommendations derived from prospective studies were indicated as level of evidence (LOE) 2a (evidence obtained from at least one well-designed controlled study without randomisation). Consensus statements based on expert opinions were indicated as LOE 4.

The SpaceOAR® System (resulting in $10 \mathrm{~mL}$ hydrogel) is FDA cleared and CE Mark approved, and commercially available in the US and most countries of Western Europe.

\section{Results}

The following key questions were developed with regard to practical aspects of hydrogel application and patient management:

1. Indication: what criteria are required to recommend the injection of a hydrogel spacer in an individual patient?

2. Injection technique: how should the injection be optimally applied?

3. Potential toxicity: which side effects could theoretically occur? 
4. Prophylaxis: are prophylactic procedures reasonable?

5. Actual toxicity: what is the current grade 2 or higher toxicity rate of hydrogel injection measured according to CTC v 4.0. ${ }^{12}$

6. Treatment of side effects: how should side effects be treated?

7. Absolute exclusion criteria: what are absolute exclusion criteria for the injection?

8. Relative exclusion criteria: what are relative exclusion criteria for the injection?

9. Special aspects of radiation treatment planning: Which aspects of radiation treatment planning should be considered?

The following key answers were developed:

\section{Indication}

A hydrogel spacer can be considered for doseescalated radiotherapy (radiation doses $\geq 76 \mathrm{~Gy}$ in conventional 1.8 - 2.0 Gy fractions) for histologically confirmed low or intermediate risk prostate cancer (LOE 2a).

A hydrogel spacer can be considered for doseescalated radiotherapy (radiation doses $\geq 76$ Gy in conventional 1.8 - 2.0 Gy fractions) for histologically confirmed prostate cancer with any localized disease (LOE 4). The risk of a microscopic T3 stage with risk of adhesions potentially impairing the hydrodissection should be considered.

Following hydrogel injection, other forms of dose-escalated radiotherapy as hypofractionated radiotherapy, particle beam radiotherapy or brachytherapy were also carried out. ${ }^{13-17}$

\section{Injection technique}

Hydrogel injection can be performed under local (possibly additional sedation), spinal or general anaesthesia. Additional procedures that are planned at the same time (i.e. brachytherapy, marker implantation etc.) determine the selected anaesthesia and should be performed in advance or a few days later since hydrogel injection might worsen visibility by air contamination. For preparation of the patient see also 3. Prophylaxis. Generally, the patient is placed in the lithotomy position. The injection is performed transperineally under transrectal ultrasound (TRUS) guidance using a linear side-fire TRUS probe and a stand-off balloon to optimize visibility. A stepper unit stabilizes the probe, so that both hands are free for the procedure.,18 The transperineal route is well known for procedures such as prostate biopsies, fiducial placement or prostate brachytherapy. ${ }^{19}$
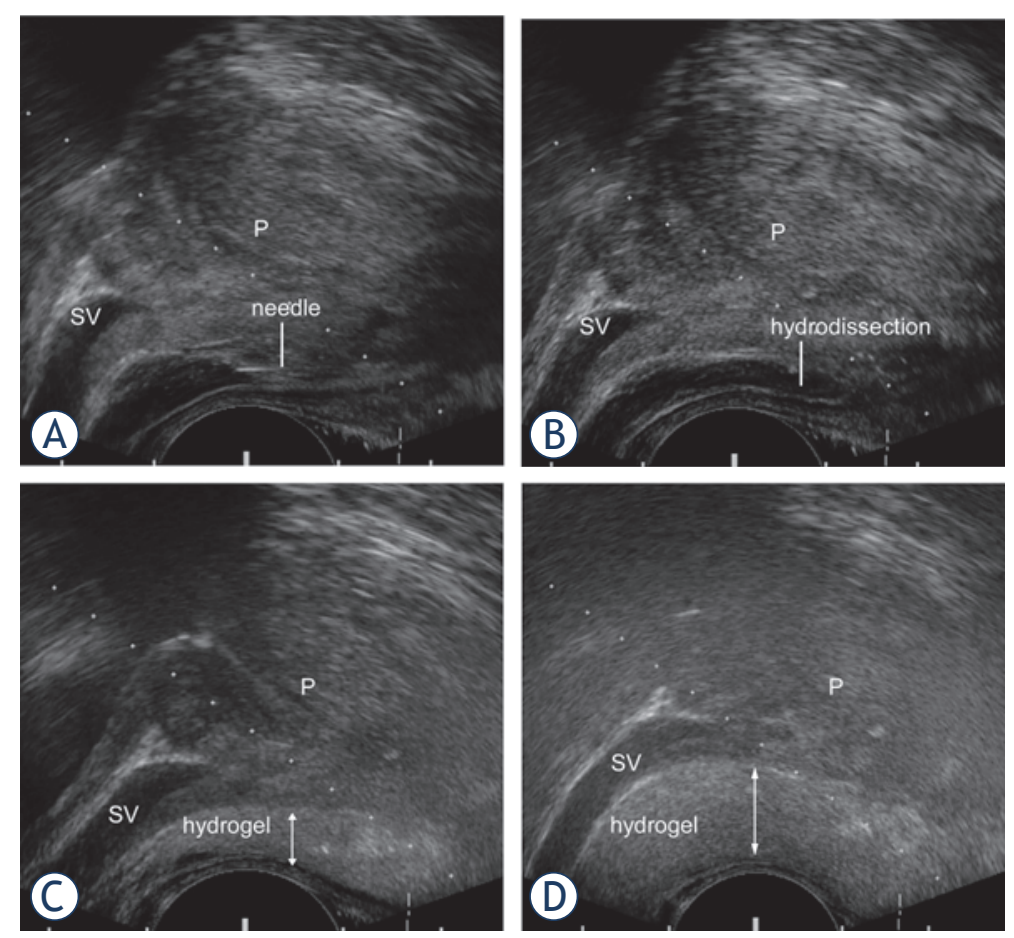

FIGURE 1. Hydrogel injection. Sagittal transrectal ultrasound images showing (A) the needle placed at the Denonvillier's fascia at the start of hydrodissection, after complete hydrodissection (B), at the start (C) and after successful hydrogel injection (D). Air contamination after hydrogel injection worsens visibility (D).

$\mathrm{P}=$ prostate $; \mathrm{SV}=$ seminal vesicles

All centres involved in this consensus used the hydrodissection technique before spacer injection to separate Denonvilliers' fascia and the anterior rectal wall. This fluid-mediated tissue separation technique is also used in other settings like cataract surgery and carpal tunnel syndrome treatment. ${ }^{20,21}$ In short, an 18 gauge needle is inserted $1-2 \mathrm{~cm}$ above the patient's anus through his perineum. The needle is advanced either parallel to the probe or slightly angled towards the prostate apex. The correct needle position is below the prostatic apex in midaxial and midsagital position of prostate (so called midgland position). Lowering the probe before hydrodissection might facilitate the procedure. Hydrodissection is performed with $10-20 \mathrm{ml}$ of saline or lidocaine (as local anaesthesia) diluted in saline. A slow injection of the fluid is necessary to ensure later a symmetric distribution of the spacer. Only in case of a successful hydrodissection, the hydrogel can be applied. 
Hydrogel is formed during the simultaneous injection i.e. mixing of the precursor (polyethylene glycol powder) and accelerator solutions (diluent). The solutions are mixed as they pass through a Y-connector prior to passing through the injection needle. Both solutions polymerise to a soft PEG-based gel within 10 seconds. An injection of $10 \mathrm{~mL}$ hydrogel results in a separation of about $9-10 \mathrm{~mm}$ between the prostate and rectal wall (Figure 1). ${ }^{6}$ The injection procedure can be completed within a few minutes.

\section{Potential toxicity}

Depending on the type of and experience with anaesthesia, patients might experience pain and discomfort during needle insertion and hydrogel injection. After spacer injection, patients may feel discomfort and rectal tenesmus. Data on pain frequency and pain intensity after injection was not routinely collected. Therefore, only retrospective data on pain management indicating the use of ampyrone sulfonate analgesics (metamizole) for the day of the procedure and sometimes afterwards was available. During spacer injection, there might be a risk of the needle and hydrogel penetrating the rectal wall, urethra, bladder or prostate. Bleeding, necrosis or ulceration of the bladder or rectal wall may follow. Lower urinary tract symptoms or even urinary retention could result from pressure on the prostate or the bladder from the spacing gel. Local inflammation or infection is possible, as with every invasive procedure. Air or hydrogel might be potentially injected into vessels.

\section{Prophylaxis}

Anticoagulants should be discontinued. Antibiotic prophylaxis is applied in some centres with fluoroquinolones or cephalosporines. However, no infections have been diagnosed up to now, even in centres with $>100$ hydrogel injections without antibiotics. A rectal enema might be used to optimize TRUS conditions during the procedure. Constipation and hard stools need to be avoided during treatment to decrease pressure on the rectal wall and a low residue diet and/or laxatives may be indicated.

\section{Actual toxicity}

Experience from all centres were participating in this consensus statement included 258 cases of hydrogel application before external beam radiotherapy for localized prostate cancer. All patients were treated with photons.
Hydrogel associated complications, defined as grade 2 or higher toxicity, were experienced by 5 patients (2\%). Hydrogel was injected intraprostatically in one single case. In 4 cases, rectum penetration was diagnosed at different intervals following injection. An injection into the rectal wall was observed in a single patient shortly after injection and radiotherapy was therefore started several weeks later. Two rectum penetrations were diagnosed during an external beam photon treatment after reports of passing mucous discharge. The patients were treated conservatively and radiotherapy was interrupted in one case. One patient reported increased bowel urgency 3-4 weeks after the end of radiotherapy before the diagnosis of a rectum penetration on proctoscopy.

All patients with the mentioned complications were followed-up with proctoscopies and/or pelvic MRI (magnetic resonance imaging). Rectal wall defects healed in all patients completely after several weeks.

\section{Observation and treatment of side effects}

Post-injection care comprises usually the first day with examination of potential urological side effects (bleeding, obstruction, pain) including the removal of a urinary catheter (if present). Side effects must be treated symptomatically. Urinary catheterization is needed in cases of urinary obstruction. Hydrogel (PEG) is not toxic or allergenic and all known injections into the prostate, bladder or rectal wall resolved without further sequelae. ${ }^{6}$ Patience is required as the hydrogel remains stable for three months and subsequently liquefies within 6 months. This was documented in $98 \%$ of patients ( $n=43 / 44)$ in the multi-center study. ${ }^{10}$ Antibiotic treatment is indicated in cases of penetration, perforation or ulceration of the rectal wall and depending on the extent, patients could be kept on parenteral nutrition or a low residue diet.

Radiotherapy should not be started during an infection or after inadvertent injection into the bladder or rectal wall before the healing process of a defect is complete.

\footnotetext{
Absolute exclusion criteria

(complication risk exceeds potential benefits)

locally advanced prostate cancer (space cannot be effectively created, tumour cell dissemination cannot be excluded)

active bleeding disorder or clinically significant coagulopathy
} 


\section{Relative exclusion criteria}

anticoagulants (discontinuation usually possible)

active inflammatory or infectious disease in the perineum or injection area (prostatitis, anorectal inflammatory disease with increased risk of ulceration, fistula or bleeding such as ulcerative colitis or Crohn's disease)

previous treatment of prostate with high risk of adhesions (high-intensity focused ultrasound, cryotherapy, radiotherapy).

Presently, very limited experience exists in hydrogel application after previous radiotherapy or high-intensity focused ultrasound. ${ }^{16,22}$ Hydrogel injection was performed without problems; however adhesions can make an injection difficult or impossible.

\section{Special aspects of radiation treatment planning}

Radiation treatment planning CT should start approximately five days after hydrogel injection to allow for decreasing of post-procedural swelling (and not to overestimate prostate volume). ${ }^{22}$ An post-injection MRI (T2-sequence sufficient without contrast media) fused to the planning CT could help to better identify the spacer (because the hydrogel is sometimes not distinguishable from the rectal wall due to same density in $\mathrm{CT}$ ). An additional advantage of an MRI is the capability to evaluate the properness of injection. Circumferential CTV-PTV-margins depend on the verification strategy (with IGRT usually $7-10 \mathrm{~mm}$, posterior if necessary less). Monitoring of the spacer volume is not necessary during radiation treatment. Stability over 3 months after injection was shown for the gel in the multicenter study. ${ }^{10}$

\section{Discussion}

The most relevant practical aspects of hydrogel injection after 258 applications were summarized in this consensus statement. A detailed description of indications, prophylaxis and management of side effects should provide new users with a fast and comprehensive introduction to the successful application of this new method. After a short learning period, the procedure can be performed to a high standard, ensuring low toxicity. Most data used are derived from well-defined controlled but not randomized studies or prospective investigations, leading to Level IIA evidence for indication and application of the hydrogel spacer.
In the multi-institutional phase II trial (52 patients recruited, 49 patients after successful spacer injection), patients were informed of higher probability of grade $2+$ toxicity, as no experience existed. With a carefully estimated probability of $6-20 \%$, it included an injection into the rectal wall, bladder wall and urethra, ulceration and necrosis of the rectal wall, bleeding and urinary retention. Three patients who were initially treated within this study experienced procedure-related events after hydrogel injection including focal rectal necrosis due to inadvertent injection of hydrogel into the rectal wall, bladder piercing during injection with hydrogel leak into the bladder, urinary retention and a device-related proctitis. ${ }^{6}$ All of these events occurred during the initial experience (learning curve in the first patient cohort) and resolved completely. Adaptations of the injection procedure (side-fire TRUS probe, stepper, stand-off balloon) were conducted which facilitated handling of the needle and hydrogel insertion. A learning curve has been reported for the application and treatment with a hydrogel, again stressing several technical aspects to achieve homogenous hydrogel distribution. This report summarizes important issues that need to be considered to achieve satisfactory spacer distribution.

Radiotherapy planning should not include the usual objectives for the dose to the rectal wall. A dose of 70 Gy can be allowed for $20 \%$ of the rectal wall volume according to RTOG (Radiation Therapy Oncology Group) recommendations. ${ }^{23}$ With a prescription dose of 76-78Gy, mean rectum volumes within the 70Gy isodose can range by about $1 \%$ with good spacer placement and adequate treatment planning. ${ }^{7}$

However, the findings of this multi-institutional evaluation of spacer-related toxicity (no G3+ event) were based on conventional fractionated doseescalated IGRT and cannot be simply adopted to other radiation treatment schedules (hypofractionation) or treatment with other ionizing radiation sources. In a study with hypofractionation using particle beam therapy (without CT-image guidance) two cases $(2 / 92 ; 2 \%)$ of G3-toxicity (colostomy) occurred, a relation to the hydrogel spacer injection cannot be excluded. ${ }^{24}$

Therefore, it is extremely important that patients are closely followed up at their centre after hydrogel injection. As the hydrogel is not tissue-toxic or allergenic, conservative management in case of inaccurate injection should be initiated as described above. Patience is required in case of inadvertent injection to the rectum or bladder wall, or in case 
of rectal wall penetration or ulceration. All cases in this analysis where this occurred healed without long-term sequelae. A currently published randomized trial demonstrated well toleration of spacer application $(10 \%$ mild transient procedural perineal discomfort) in 149 patients suggesting safety of this method with conventional fractionated doseescalated IGRT, too. ${ }^{25}$

For optimized injection results, one expert in each centre was trained by another expert. The procedure was performed by only one or two experts at each centre, guaranteeing a high degree of experience. Last but not least, correct patient selection is essential. The optimal patient for this new method is at low risk of adhesions (inflammation, tumour spread due to locally advanced disease) and has a low risk of bleeding. The risk of tumour displacement by hydrodissection is very small, since prostatectomy series with limited pT3 stages reported in less than one fifth of patients an invasion and in no case a progression through the full thickness of the Denonvilliers' fascia. ${ }^{26}$

After successful injection, the benefit for the patients was measured by acute toxicity scores and by radiation planning parameters (dose-volume histograms). In brief, the theoretical benefit of an additional space between prostate and rectum translated into improved radiation treatment plans with approximately $10 \%$ reduction in relevant high-dose areas (dose level from 40-70Gy). ${ }^{8}$ These improved radiation treatment plans with lower rectal doses converted into reduced acute toxicity rates. Grade 2 proctitis resulting from radiotherapy was a rare event compared to standard conformal or intensity-modulated radiotherapy, for example $12.5 \%$ acute toxicity in the multicenter phase II trial $^{10}$ in comparison to occasionally $50 \%$ or more in studies without a spacer. ${ }^{27,28}$ The prevention of acute proctitis with this procedure is a benefit for the patient. Further benefits for the patients are conceivable. Consequential late side effects derive from persisting acute toxicity ${ }^{29}$ and reduced acute toxicity will usually be associated with a lower risk of late toxicity. However, the evaluation of this potential long-term benefit needs longer follow-up. Another beneficial effect of improved rectum protection is the facilitation of dose escalation to the prostate. Since increased radiation doses improve outcome in the range of approximately $1.5 \%$ better biochemical control per Gy after a mean follow-up of five years ${ }^{1}$, these dosimetric changes are relevant for improved tumour control with a lower risk of toxicity.
This spacer consensus focuses on the use of Polyethylene-glycol (PEG) hydrogel spacers in dose-escalated radiotherapy of prostate cancer. However, at least four different bio-resorbable spacer materials (PEG-hydrogel, balloon of copolymer of polylactic acid or similar poly ( $\alpha$-hydroxy acids), hyaluronic acid and collagen) are currently evaluated. PEG hydrogel spacers and bio-resorbable balloons have demonstrated an excellent biocompatibility profile in humans compared to other spacers made of hyaluronic acid or collagen. ${ }^{30}$ Direct comparison of PEG hydrogel spacer and bio-resorbable balloon demonstrated the following. PEG spacers were less invasive (smaller needle diameter with 1.3 vs. $2-3 \mathrm{~mm}$ ). The balloon spacer was superior in reducing rectum dose $(-28 \%)$, but exhibited an average volume loss of $>50 \%$ during the full course of treatment (37-40 fractions), while the volume of gel spacers remained fairly constant. ${ }^{31}$

Displacement of radiosensitive organs by spacers is not limited to primary prostate cancer alone. Further applications being investigated include treatment of recurrent prostate cancers ${ }^{32}$, gynecological malignancies ${ }^{33}$ and esophageal gel-shifting facilitating treatment of mediastinal nodes. ${ }^{34}$ The principle to displace radiosensitive organs from high dose areas is also used in case of adhesions of small intestinum and radiation targets. For such special situations are invasive surgical techniques available like laparoscopic mesh placement. ${ }^{35}$

We conclude that hydrogel injection can be considered for dose-escalated radiotherapy. Well trained physicians, correct patient selection and knowledge of the management of potential side effects are essential for optimal application. The benefit for the patient is improved protection of the rectal wall, which is associated with low radiation related proctitis rates. This allows dose-escalation associated with improved tumour control. However, a very low rate of a potential serious adverse event cannot be excluded and should carefully be discussed with the patient and be balanced against potential benefits. The evaluation of this potential long-term benefit needs longer follow-up.

\section{Disclosures}

The consensus meeting was supported by CSDiagnostics, Neuss, Germany. Augmenix, Inc., Waltham, Massachusetts, United States is the sponsor of the multicentre-study and sponsored with 
CSDiagnostics, Neuss, Germany additional hydrogels for patients treated outside the multicentre study. TK reports grants from CSDiagnostics, Neuss, Germany for another meeting.

\section{Acknowledgment}

Dr. Elizabeth Krämer performed the copyediting. We acknowledge support by Deutsche Forschungsgemeinschaft (DFG) and Open Access Publishing Fund of University of Tübingen.

\section{References}

1. Viani GA, Stefano EJ, Afonso SL. Higher-than-conventional radiation doses in localized prostate cancer treatment: a meta-analysis of randomized, controlled trials. Int J Radiat Oncol Biol Phys 2009; 74: 1405-18.

2. Dearnaley D, Syndikus I, Sumo G, Bidmead M, Bloomfield D, Clark C, et al. Conventional versus hypofractionated high-dose intensity-modulated radiotherapy for prostate cancer: preliminary safety results from the $\mathrm{CHHiP}$ randomised controlled trial. Lancet Oncol 2012; 13: 43-54.

3. Hatiboglu G, Pinkawa M, Vallee JP, Hadaschik B, Hohenfellner M. Application technique: placement of a prostate-rectum spacer in men undergoing prostate radiation therapy. BJU Int 2012; 110: E647-52.

4. Eckert F, Alloussi S, Paulsen F, Bamberg M, Zips D, Spillner P, et al. Prospective evaluation of a hydrogel spacer for rectal separation in doseescalated intensity-modulated radiotherapy for clinically localized prostate cancer. BMC Cancer 2013; 13: 27.

5. Pinkawa M, Piroth MD, Holy R, Escobar-Corral N, Caffaro M, Djukic V, et al. Spacer stability and prostate position variability during radiotherapy for prostate cancer applying a hydrogel to protect the rectal wall. Radiother Oncol 2013; 106: 220-4

6. Uhl M, van Triest B, Eble MJ, Weber DC, Herfarth K, De Weese TL. Low rectal toxicity after dose escalated IMRT treatment of prostate cancer using an absorbable hydrogel for increasing and maintaining space between the rectum and prostate: results of a multi-institutional phase II trial. Radiother Oncol 2013; 106: 215-19.

7. Pinkawa M, Klotz J, Djukic V, Schubert C, Escobar-Corral N, Caffaro M, et al. Learning curve in the application of a hydrogel spacer to protect the rectal wall during radiotherapy of localized prostate cancer. Urology 2013; 82: 963-8.

8. Song DY, Herfarth KK, Uhl M, Eble MJ, Pinkawa M, van Triest B, et al. A multiinstitutional clinical trial of rectal dose reduction via injected polyethyleneglycol hydrogel during intensity modulated radiation therapy for prostate cancer: analysis of dosimetric outcomes. Int J Radiat Oncol Biol Phys 2013; 87: $81-7$.

9. Pinkawa M, Piroth MD, Holy R, Escobar-Corral N, Caffaro M, Djukic V, et al. Quality of life after intensity-modulated radiotherapy for prostate cance with a hydrogel spacer. Matched-pair analysis. Strahlenther Onkol 2012 188: $917-25$

10. Uhl M, Herfarth K, Eble MJ, Pinkawa M, van Triest B, Kalisvaart R, et al. Absorbable hydrogel spacer use in men undergoing prostate cance radiotherapy: 12 month toxicity and proctoscopy results of a prospective multicenter phase II trial. Radiat Oncol 2014; 9: 96

11. Klotz T, Mathers MJ, Lazar Y, Gagel B. Use of hydrogel as spacer in Denovier's space: optimization of IMRT radiotherapy of localized prostate cancer. Urologe A 2013; 52: 1690-97.

12. National Cancer Institute. Common terminology criteria for adverse events v.4.0 (CTCAE). 2011. [citated 14 Feb 2016]. Available from: http://ctep.cancer.gov/protocolDevelopment/electronic applications/ctc.htm.
13. Beydoun N, Bucci JA, Chin YS, Malouf D, Enari E, Painter SD. First report of transperineal polyethylene glycol hydrogel spacer use to curtail rectal radiation dose after permanent iodine- 125 prostate brachytherapy. Brachytherapy 2013; 12: 368-74.

14. Christodouleas JP, Tang S, Susil RC, McNutt TR, Song DY, Bekelman J, et al. The effect of anterior proton beams in the setting of a prostate-rectum spacer. Med Dosim 2013; 38: 315-9.

15. Habl G, Hatiboglu G, Edler L, Uhl M, Krause S, Roethke M, et al. Ion Prostate Irradiation (IPI) - a pilot study to establish the safety and feasibility of primary hypofractionated irradiation of the prostate with protons and carbon ions in a raster scan technique. BMC Cancer 2014; 14: 202.

16. Nguyen PL, Devlin PM, Beard CJ, Orio PF, 3rd, O'Leary MP, Wolfsberger LD, et al. High-dose-rate brachytherapy for prostate cancer in a previously radiated patient with polyethylene glycol hydrogel spacing to reduce rectal dose: case report and review of the literature. Brachytherapy 2013; 12: 77-83.

17. Rucinski A, Bauer J, Campbell P, Brons S, Unholtz D, Habl G, et al. Preclinical investigations towards the first spacer gel application in prostate cancer treatment during particle therapy at HIT. Radiat Oncol 2013; 8: 134.

18. Pinkawa M. Spacer application for prostate cancer radiation therapy. Future Oncol 2014; 10: 851-64.

19. Ng M, Brown E, Williams A, Chao M, Lawrentschuk N, Chee R. Fiducial markers and spacers in prostate radiotherapy: current applications. BJU Int 2014; 113 (Suppl 2): 13-20.

20. Malone D, Clark T, Wei N. Ultrasound-guided percutaneous injection, hydrodissection, and fenestration for carpal tunnel syndrome: description of a new technique. J Appl Res 2010; 10: 116-23.

21. Vasavada AR, Singh R, Apple DJ, Trivedi RH, Pandey SK, Werner L. Effect of hydrodissection on intraoperative performance: randomized study. $J$ Cataract Refract Surg 2002; 28: 1623-8.

22. Pinkawa M, Bornemann C, Escobar-Corral N, Piroth MD, Holy R, Eble MJ. Treatment planning after hydrogel injection during radiotherapy of prostate cancer. Strahlenther Onkol 2013; 189: 796-800.

23. Lawton CA, Michalski J, El-Naqa I, Buyyounouski MK, Lee WR, Menard C, et al. RTOG GU Radiation oncology specialists reach consensus on pelvic lymph node volumes for high-risk prostate cancer. Int J Radiat Oncol Biol Phys 2009; 74: 383-7.

24. Habl G, Uhl M, Hatiboglu G, Ellerbrock M, Debus J, Herfarth K. Hypofractionated radiotherapy of the prostate using carbon ions or protons - acute toxicities of the IPI-study. [German], [Abstract]. 20th Annual Congress of the German Society for Radiation Oncology. Dusseldorf, Germany; Jul 3-6, 2014. Strahlenther Onkol 2014; 190(Suppl 1): 136

25. Mariados N, Sylvester J, Shah D, Karsh L, Hudes R, Beyer D, et al. Hydrogel Spacer Prospective Multicenter Randomized Controlled Pivotal Trial: Dosimetric and Clinical Effects of Perirectal Spacer Application in Men Undergoing Prostate Image Guided Intensity Modulated Radiation Therapy. Int J Radiat Oncol Biol Phys 2015; 92: 971-7.

26. Villers A, McNeal JE, Freiha FS, Boccon-Gibod L, Stamey TA. Invasion of Denonvilliers' fascia in radical prostatectomy specimens. J Urol 1993; 149: 793-8.

27. Vora SA, Wong WW, Schild SE, Ezzell GA, Halyard MY. Analysis of biochemical control and prognostic factors in patients treated with either low-dose three-dimensional conformal radiation therapy or high-dose intensitymodulated radiotherapy for localized prostate cancer. Int J Radiat Oncol Biol Phys 2007; 68: 1053-8.

28. Zietman AL, Bae K, Slater JD, Shipley WU, Efstathiou JA, Coen JJ, et al. Randomized trial comparing conventional-dose with high-dose conformal radiation therapy in early-stage adenocarcinoma of the prostate: long-term results from proton radiation oncology group/american college of radiology 95-09. J Clin Oncol 2010; 28: 1106-11.

29. Pinkawa M, Holy R, Piroth MD, Fischedick K, Schaar S, Szekely-Orban D, et al. Consequential late effects after radiotherapy for prostate cancer - a prospective longitudinal quality of life study. Radiat Oncol 2010; 5: 27.

30. Mok G, Benz E, Vallee JP, Miralbell R, Zilli T. Optimization of radiation therapy techniques for prostate cancer with prostate-rectum spacers: a systematic review. Int J Radiat Oncol Biol Phys 2014; 90: 278-88. 
31. Wolf F, Gaisberger C, Ziegler I, Krenn E, Scherer P, Hruby S, et al. Comparison of two different rectal spacers in prostate cancer external beam radiotherapy in terms of rectal sparing and volume consistency. Radiother Oncol 2015; 116: 221-5.

32. Arcangeli S, Agolli L, Donato V. Retreatment for prostate cancer with stereotactic body radiation therapy (SBRT): Feasible or foolhardy? Rep Pract Oncol Radiother 2015; 20: 425-9.

33. Viswanathan AN, Damato AL, Nguyen PL Novel use of a hydrogel spacer permits reirradiation in otherwise incurable recurrent gynecologic cancers. J Clin Oncol 2013; 31: e446-7.

34. Kishi K, lida T, Ojima T, Sonomura T, Shirai S, Nakai M, et al. Esophageal gel-shifting technique facilitating eradicative boost or reirradiation to upper mediastinal targets of recurrent nerve lymph node without damaging esophagus. J Radiat Res 2013; 54: 748-54.

35. Bachmann R, Heinzelmann F, Muller AC, Ladurner R, Schneider CC, Konigsrainer A, et al. Laparoscopic pelvic mesh placement with closure of pelvic floor entrance to prevent small intestine radiation trauma - A retrospective cohort analysis. Int J Surg 2015; 23: 62-7. 